\title{
EL PAPEL DE RODRIGO FACIO EN LA CONSTITUYENTE DE 1949
}

\section{THE ROLE OF RODRIGO FACIO IN THE CONSTITUENT OF 1949}

\author{
Alex Solís Fallas*
}

\author{
Una Constitución se hace para un pueblo \\ con puntos de vista fundamentalmente diferentes. \\ Rodrigo Facio
}

\begin{abstract}
RESUMEN
Rodrigo Facio fue la figura estelar y el principal ideólogo de la Asamblea Nacional Constituyente de 1949. El Estado democrático y social derecho concebido en la Constitución que nos rige, es producto, principalmente de sus ideas políticas y luchas en la Constituyente. Se dice con énfasis, hoy, cuando la moda de nuestros líderes políticos es renegar de la ideología y decir que se es de centro, a pesar de que eso conduzca, nada más, que al estancamiento político, económico y social del país.

El pensamiento de Rodrigo Facio se reviste de una particular actualidad en momentos cuando la democracia costarricense, como diría O' Donnell, no tiene un buen desempeño. La opinión generalizada es que los últimos gobiernos, dominados por el personalismo y el egocentrismo, han perdido el rumbo; no se están solucionado cuestiones básicas del desarrollo, como la pobreza y la desigualdad social; tampoco ha encarado, con valentía e ingenio, el problema relacionado con la inseguridad ciudadana, producto tanto de la criminalidad común como del lavado de dólares, el narcotráfico y el sicariato.
\end{abstract}

PALABRAS CLAVE: RODRIGO FACIO * REVOLUCIÓN * ASAMBLEA NACIONAL CONSTITUYENTE * CONSTITUCIÓN POLÍTICA * IDEÓLOGO * ESTUDIOSO * JUSTICIA SOCIAL *DIGNIDAD HUMANA

\section{ABSTRACT}

Rodrigo Facio was the star figure and the main ideologist of the National Constituent Assembly of 1949. The Democratic state and the law social conceived in the Constitution that governs us is the result, primarily, of his political ideas and struggles in the Constituent Assembly. I say with emphasis, today, when the fashion of our political leaders is to renege about the ideology and say that is of center, although it leads, nothing more, that to the political, economic and social stalemate in the country.

Rodrigo Faciós thought has a particular actuality at a time when the Costa Rican democracy, as O' Donnell would say, it hasn't a good performance. The general opinion is that

Instituto de Investigaciones Jurídicas (IIJ) de la Facultad de Derecho de la Universidad de Costa Rica (UCR). alexsolic@racsa.co.cr 
the last governments, dominated by personalism and egocentrism, have lost their way, they are not solved basic issues of the development such as the poverty and the social inequality; they neither have faced, with courage and wit, to the problem related to insecurity, product of the common crime and the money laundering, drug trafficking and contract killings.

KEYWORDS: RODRIGO FACIO * REVOLUTION * NATIONAL CONSTITUENT ASSEMBLY * POLITICAL CONSTITUTION * IDEOLOGIST * STUDIOUS * SOCIAL JUSTICE * HUMAN DIGNITY

\section{INTRODUCCIÓN ${ }^{1}$}

En los tiempos que corren, se acepta, de manera generalizada, pacífica y con mucha admiración y respeto, que Rodrigo Facio Brenes fue "una de las más deslumbrantes figuras del escenario intelectual y político de la Costa Rica de mediados del Siglo Xx" (Monge, 2003: xv). No en vano, esta casa de estudios lleva su nombre; $y$ no nos debe extrañar que unos y otros reconozcan a Rodrigo Facio como el principal personaje de la Asamblea Nacional Constituyente de 1949. Precisamente, esta mañana, se me ha invitado para que explique "¿cuál fue su papel en la Asamblea Nacional Constituyente de 1949?”.

Para lo anterior, podría agruparse la información de varias maneras. En lo personal, sería muy cómodo limitarme a narrar, contar, relatar y enumerar lo que hizo Rodrigo Facio, para justificar porqué se le considera el personaje estelar de aquel gran reparto, en la Constituyente de 1949. No obstante lo anterior, en lugar de discurrir por ese camino plano y seguro, prefiero ir por otras rutas más escabrosas y peligrosas, quizás también, más polémicas y controvertidas. No quiero de manera pasiva contar una historia; intento un análisis, a partir de ciertos conceptos $y$

$1 \quad$ Esta conferencia fue elaborada con fundamento en las intervenciones que realizó Rodrigo Facio Brenes, en la Asamblea Nacional Constituyente, que en 1949, proclamó la Constitución que hoy nos rige. Todos sus discursos fueron recopilados por Oscar Castro Vega, quien fuera el Secretario de aquella Asamblea Constituyente. Por eso, a lo largo de la conferencia se cita constantemente su libro: Rodrigo Facio en la Constituyente de 1949, editado por EUNED, en el año 2003. Por tanto, prácticamente, todas las citas están tomadas de las propias palabras de Rodrigo Facio. categorías; advierto, en consecuencia, que soy selectivo en cuanto a los episodios que estudio y la interpretación que les doy.

Me propongo interrogar el papel histórico de Rodrigo Facio desde el punto de vista de la ética política imperante, ciertamente, para aprender de él, pero también para enjuiciar la forma como quienes nos gobiernan están abordando la solución de los problemas políticos, económicos, sociales y culturales que aquejan a nuestro país. La comparación tiene sentido, porque los problemas que Rodrigo Facio denunció en 1949 son básicamente los mismos que hoy aquejan a la sociedad costarricense: la falta de liderazgo, el personalismo, la intransigencia, el dogmatismo mesiánico, el fraude, la mentira, el chantaje y la política del miedo.

Mi hipótesis es que Rodrigo Facio fue un hombre ejemplar en su vida pública y privada, vida que deberíamos emular los ciudadanos, los profesores y la clase política, tan venida a menos en los últimos tiempos.

A veces estudiamos la historia para evitar los errores en que hemos incurrido como sociedad, hoy propongo lo contrario, que estudiemos a Rodrigo Facio para emularlo y de esta manera, corregir lo que estamos haciendo mal como país, en los tiempos que corren.

Hay mucho material para ello. Revisando las Actas de la Asamblea Nacional Constituyente, uno se encuentra, que casi no hubo un tema sobre el cual no se pronunciara el Constituyente Facio en la discusión de lo que sería la nueva Costa Rica. Hay intervenciones verdaderamente épicas, como aquella en la que defiende el Proyecto de Constitución impulsado por la Junta de Gobierno o en la que explica cómo han de ser las relaciones entre un gobierno de facto, que surge de una revolución y una Asamblea 
Nacional Constituyente; o aquella en la que aborda el espinoso tema de la función social de la propiedad privada, o en la que defiende las garantías sociales, o las instituciones autónomas, o el financiamiento permanente del presupuesto de la Universidad de Costa Rica, para citar algunos ejemplos.

\section{EL ESTUDIOSO}

Antes de proseguir, para efectos de contextualizar lo que se viene explicando, se debe recordar que después de la Guerra Civil, la Junta Fundadora de la Segunda República presidida por José Figueres Ferrer, nombró una comisión especial integrada por nueve personas, que se constituyó el 25 de mayo de 1949 , con el fin de que redactara un proyecto de Constitución, para que sirviera como la base para las discusiones a la Asamblea Nacional Constituyente, que se instalaría el 15 de enero de 1949. Rodrigo Facio fue el Secretario de esa comisión y se dice, uno de los principales artífices del proyecto de Constitución (Castro, 2003: 3).

Este hecho, que podría pasar por lo puramente anecdótico, tiene en Rodrigo Facio, una especial significación: consciente de la grave misión que le ha sido encomendada, estudia de manera incansable, con muchos meses de anticipación, hasta muy altas horas de la noche, en la elaboración del Proyecto de Constitución.

Para ello, primero hace un diagnóstico de la realidad nacional, como explicará en una de sus disertaciones, ya siendo constituyente. Ahí sugiere que se debe trasformar la política tradicional, dominada por partidos personalistas, sin rumbo, sin ideología y acostumbrados a gobernar al margen de la Constitución. Ataca al Poder Ejecutivo al que considera hipertrofiado $y$ omni-decisivo, con poderes casi absolutos sobre los empleados públicos, las finanzas, los ministros, el Congreso, el proceso electoral, el Poder Judicial y las municipalidades (Castro, 2003: 75). De ese personalismo que ha relegado lo institucional a un segundo plano, decía Rodrigo Facio, "derivan las candidaturas oficiales, el relajamiento de la burocracia, el servilismo y el arrebañamiento de los Congresos..." (Castro, 2003: 120). En su diagnóstico, Rodrigo
Facio señala que la influencia oficial y la politiquería han sido de tal magnitud, que se ha ido:

... formando una fauna de políticos profesionales que expulsaron de la escena nacional a los verdaderos valores [Luego el Constituyente Facio sentencia:] Cuando un pueblo elige presidente a un hombre sin ideas, sin record de estadista, sin planes... solo porque es muy bueno, algo debe andar mal en ese país. Algo debe estar descompuesto. Alguna falla grave debe estar carcomiendo la estructura de la nacionalidad (Castro, 2003: 75) [o a una mujer diría yo, hoy, aquí].

Por cierto, quisiera advertir que con esa descripción, Rodrigo Facio solo se refiere a los hechos que desembocaron en la Guerra Civil de 1948, cualquier similitud con algunas cosas que están pasando ahora, es pura casualidad.

En ese análisis, Facio concluye diciendo que la Constitución de 1871, había carecido de fuerza normativa real y efectiva, predominando el personalismo. Por tanto sentencia, se debe promulgar una Constitución que racionalice el personalismo, que limite el ejercicio del poder político, que regule la acción pública y termine con la politiquería (Castro, 2003: 74).

En segundo término, en ese esfuerzo de preparación, Rodrigo Facio, en su fase de historiador hace un estudio detallado de nuestra historia constitucional; en su fase de abogado, analiza desde la perspectiva del derecho comparado, la jurisprudencia y las constituciones vigentes en muchísimos países del mundo y en la de economista, para justificar el intervencionismo estatal, explica con exquisita claridad las políticas económicas imperantes, entre otros países, en Estados Unidos, Inglaterra y Francia.

Por último, solo por citar un ejemplo más de la seriedad y responsabilidad con que se preparó nuestro personaje para ser Constituyente, hay que citar que en su esfuerzo investigativo, estudió filosofía del derecho; en sus discursos cita autores que, todavía hoy estudiamos en la Facultad de Derecho, autores de la talla de Oliver Wendell Holmes, Herman 
Heller, Hans Kelsen y Luis Recaséns Siches. Hay que enfatizar, que con este último intercambió correspondencia, con el fin de aclarar $y$ profundizar sobre las facultades del poder revolucionario y el poder constituyente, para luego concretar la naturaleza de las relaciones que debía existir entre la Junta de Gobierno, presidida por José Figueres Ferrer y la Asamblea Nacional Constituyente, instalada para dotar a Costa Rica de una nueva Constitución.

\section{EL ESTRATEGA}

Para comprender la figura de Rodrigo Facio, tenemos que recordar que la Asamblea Nacional Constituyente quedó integrada por 45 legisladores constituyentes, de los cuales 34 pertenecían al Partido Unión Nacional, 6 al Partido Constitucional y solo 4 al Partido Social Demócrata: Rodrigo Facio, Luis Alberto Monge Álvarez, Rogelio Valverde y Fernando Fournier. Quiero hacer énfasis en que el Partido Social Demócrata era una minoría insignificante, frente a los otros 41 diputados - los unionistas y constitucionalistas- representantes de los poderosos grupos cafetaleros y oligárquicos, además, furibundos enemigos de José Figueres y la Junta de Gobierno.

En ese contexto de minoría, el Proyecto de Constitución elaborado por la Junta Fundadora de la Segunda República, en el que Rodrigo Facio había trabajado por mucho tiempo y hasta altas horas de la noche, fue desechado por la constituyente del 49 como base de discusión, acogiéndose en su lugar la Constitución de 1871.

Esto significó, en aquel momento, un durísimo golpe para las aspiraciones que tenía la Junta de Gobierno y los socialdemócratas de dotar a Costa Rica con una Constitución "equilibrada" como decía el constituyente Facio, entre la "justicia social y la eficiencia económica", donde "la justicia no mate a la eficiencia, ni la eficiencia mate a la justicia" (Castro, 2003: 98).

¿Qué hizo Rodrigo Facio ante la derrota del Proyecto de Constitución?

No gritó, no vociferó, no amenazó, no trató a nadie de derechista, fascista, comunista o bandido. Desde su curul, dicen sus glosadores, habla más como estadista y catedrático universitario que como dirigente de un partido político; lo hace con sinceridad y vehemencia, pero nunca pierde la compostura (Castro, 2003: 54).

Él sabe que, desde el ámbito político, las palabras invitan a la acción; que con las palabras, además de nombrar cosas, se hacen cosas y que las palabras tienen una entidad que las trasciende $y$ sirven para perfeccionar o degradar al ser humano, para construir o para destruir la sociedad. Rodrigo Facio está consciente de que el país viene saliendo de una violenta guerra, donde la palabra se ha desangrado en pasión y violencia. Comprende, como diría Foucault, que la Constituyente es la continuación de la guerra, pero por otros medios, en la que impera la confrontación de ideas, las discusiones acaloradas y la lucha ideológica.

En ese contexto, Rodrigo Facio no reclama el resultado de la votación, porque él sabe como buen demócrata, que la ley de la mayoría se ha impuesto y que, en ese momento, nada se podía hacer; también sabe, como persona culta, que el silencio en la música también tiene su valor y que una Asamblea Constituyente no es una sala de esgrima ni un escenario para oradores histriónicos.

Es cierto que esa derrota, ese golpe, causó un gran dolor a Rodrigo Facio y sus tres compañeros constituyentes del Partido Social Demócrata. ¡Estos tres amenazan con no volver a sesiones! Los cuatro se habían preparado para discutir el texto de la futura Constitución con fundamento; el Proyecto de la Junta Fundadora de la Segunda República y de repente, sus sueños, su programa de trabajo, en fin, su estrategia se había hecho añicos.

Entonces comenzó a emerger Rodrigo Facio como estratega, hábil negociador, tolerante $y$ respetuoso de las ideas y opiniones de los otros constituyentes (Castro, 2003: 11), pero, además, como hombre perseverante y luchador. Está decidido a rescatar su Proyecto de Constitución. Y como un gran general, convence a sus compañeros que deben continuar la lucha a favor de una constitución democrática, pero utilizando otros medios. 
Para ello, rediseña la estrategia parlamentaria. Con una muestra de gran valentía e ingenio insisten en su proyecto de constitución, pero ahora de manera desagregada, es decir, en forma de mociones, dirigidas a modificar la constitución de los conservadores y protectores del statu quo. Nos imaginamos entonces, como esta nueva estrategia, a su vez, significó una bofetada para el grupo conservador. El ambiente se agitó, las estrategias se sucedían una a otra, la lucha revolucionaria continuaba pero en otro lugar: el Palacio Nacional y por otros medios (la palabra y los argumentos), terrenos en los que Rodrigo Facio era un maestro.

\section{EL REVOLUCIONARIO}

Rodrigo Facio quería cambiar lo que ocurría en el país. Su intranquilidad, su espíritu revolucionario no se cultivó con la Revolución que encabezó José Figueres. Antes de que Pepe Figueres entrara a la política, Rodrigo Facio ya había fundado el Centro para los Estudios de los Problemas Nacionales, en 1942 y el Partido Social Demócrata, en 1945.

Por ello, cuando le nombraron miembro de la Asamblea Nacional Constituyente, a los 32 años de edad, se enfrentó a la gran oportunidad o quizás a la necesidad, de formular su visión social del Estado de Derecho y por tanto, de dejar su particular huella en la historia costarricense.

En la defensa del Proyecto de Constitución de la Segunda República, pronuncia uno de sus discursos más memorables, apasionados y visionarios. Ahí nos dice:

La revolución abrió y forzó el camino para iniciar una honda transformación del país. Se presentó al fin una oportunidad, que no se encuentra con mucha frecuencia, pues faltan casi siempre los cauces para que un pueblo pueda dejar expresados sus anhelos y sus ideales de manera integral. Hoy todo el pueblo, incluyendo a sus más valiosas instituciones, está con el deseo de que Costa Rica se organice sobre bases nuevas y modernas, que sean capaces de darle al hombre medio mejores garantías para su libertad política, para su trabajo, para su familia, para su vida pública y privada (Castro, 2003: 57).

\section{ESTADO DEMOCRÁTICO}

Claro de su misión y de la visión del Estado que añora, en una sesión reflexiona "estamos haciendo una Constitución” (Castro, 2003: 252), estamos conformando un nuevo Estado de Derecho.

¿Y de qué Estado nos habla Rodrigo Facio?

Primero, contesta negativamente a esa pregunta, diciéndonos lo que no debe ser el Estado:

Adjuramos del Estado hipertrofiado, personalista, del Ejecutivo a la Prusia, de la seguridad social recetada por Bismark. [Inmediatamente, después de explicar lo que rechaza, expone una versión moderna del tipo de Estado que quiere fundar. Dice:] ... queremos un Estado democrático, libre, eficaz, responsable, controlado, dentro del cual la división de funciones y la descentralización garanticen a la ciudadanía contra presuntos irrespetos a sus derechos (Castro, 2003: 119).

De conformidad con lo anterior, resulta claro que para Rodrigo Facio, el Estado no es un fin en sí mismo. Por el contrario, como reafirmaría muchos años después la Sala Constitucional, Facio pensaba que la democracia:

... es una forma de Estado que implica una relación entre el poder y los hombres, que se resuelve de modo favorable a la dignidad de la persona, a su libertad $y$ a sus derechos ${ }^{2}$, [o como diría Harold Laski], no existo únicamente para el Estado, pero, tampoco, el Estado existe solamente para mí (1978: 7).

Uno podría decir, con Rodrigo Facio, de acuerdo al tipo de Estado que él nos legó en la

$2 \quad$ Sala Constitucional de la Corte Suprema de Justicia, voto nro. 3336-94. 
Constitución Política, que no se reconoce la dignidad de la persona, su libertad y sus derechos fundamentales para limitar al poder sino a la inversa, que se regula, limita y controla al poder político para permitir el desarrollo integral de la personalidad, porque el meollo del estado democrático y social de derecho se encuentra en la persona y no en el poder político.

Por eso dice Rodrigo Facio, vamos a darle al pueblo costarricense:

... una nueva Constitución que responda a aquellos anhelos, para que a la par de la libertad y la democracia política, se garanticen a su vez, medidas que sirvan de fundamento material para que el pueblo pueda ejercer sus derechos políticos, sin la carga de condiciones sociales $y$ económicas que violen su capacidad de vivir y su necesidad de vivir dignamente (Castro, 2003: 58).

\section{EL CONSTITUCIONALISTA}

Con esas ideas, poco a poco, Rodrigo Facio se va convirtiendo en la principal figura de aquel cuerpo Constituyente. Cuenta Óscar Castro, el Secretario de Actas de la Asamblea Nacional Constituyente, que Rodrigo Facio siempre acaparaba la atención por sus conocimientos; que tiene la singular capacidad de abordar las cuestiones más complejas y técnicas de manera clara y sencilla; $y$ que nunca improvisa sus grandes discursos, los que planea, con lujo de destalles, con días de anticipación y durante muchas horas. Está demás enfatizar, que en aquella época no habían asesores que estudiaran y escribieran por él.

Así, pues, no nos debe de extrañar que cada intervención de Rodrigo Facio sea como un soplo de aire fresco, una ventana de luz, cuyos rayos nos alumbra hasta nuestros días. En su participación en la Constituyente, es fácil adivinar cuanto disfruta de su trabajo como investigador, político, historiador, economista y jurista.

Desde el mundo jurídico, Rodrigo Facio posee el talento innato para discernir casi de inmediato el problema jurídico que se plantea y encontrar su resolución. De ahí que, cuando la
Asamblea Nacional Constituyente se pregunta qué es una Constitución Política, Rodrigo Facio aborda el tema con una claridad y sabiduría que empalidecería a los más famosos juristas de nuestro tiempo y claro está, también a nuestro famoso Tribunal Constitucional.

¿Cuál es la Constitución que imagina Rodrigo Facio?

No hay que deducirlo, él contesta de manera expresa, sin titubeos, claro de su función constituyente y de la visión del tipo de Estado que quería: "la constitución debe ser una norma de equilibrio" (Castro, 2003: 96). Porque, "una Constitución se hace para un pueblo con puntos de vista fundamentalmente diferentes" (Castro, 2003: 66).

También en otra oportunidad manifiesta:

... una buena constitución política debe ser un todo orgánico, armonioso, cuyos diferentes capítulos guarden relación lógica entre sí, y respondan a un mismo criterio fundamental sobre cuáles son los fines del Estado, $y$ cual sea la forma en que éste debe organizarse para cumplirlos mejor (Castro, 2003: 51-52).

Cuando se le cuestiona sobre el tamaño de la Constitución responde con seguridad: "El estudio del Derecho Constitucional comparado no permite precisar la extensión que debe tener una Carta Fundamental y ni siquiera las materias a que debe limitarse (Castro, 2003: 77). Ese tema pareciera no tener relevancia para Rodrigo Facio, con tal de que se resguarden bien, "los eternos principios de la libertad humana $y$ la dignidad individual" (Castro, 2003: 121). En tal sentido manifiesta, que lo que ha hecho los constituyentes del Partido Social Demócrata, "es seguir las modernas corrientes del constitucionalismo social que se están materializando en un nuevo tipo de Estado; "en un estado que se interesa por los problemas generales del ciudadano y no sólo por su problema político frente al gobierno (sic)" (Castro, 2003: 79).

\section{EL IDEÓLOGO}

La Constitución de Rodrigo Facio, digo la Constitución de 1949, hablando desde el plano 
ideológico, no es una norma neutra, en el sentido de que se pueda gobernar para cualquier fin y de cualquier forma. Cómo podría serlo, si Rodrigo Facio no se declaró neutral, ni asumió una actitud calculadora respecto a ningún tema, tal y como sucede en los tiempos que corren, con nuestra clase dirigente.

Desde ese punto de vista, la Constitución no termina siendo un conjunto de regulaciones inconexas o yuxtapuestas en un texto normativo. Por el contrario, para Facio, la Constitución representa una toma de posición valorativa, entiéndase ideológica, en favor del Estado democrático y social de derecho, donde el ser humano, su dignidad $y$ sus derechos fundamentales son el centro y el fin de toda acción pública, y el Estado consiguientemente, solo es un instrumento, un medio, una herramienta a su servicio.

En congruencia, Rodrigo Facio defiende la intervención del Estado, con el propósito de asegurarle al hombre medio no solo la libertad política, sino también la seguridad y libertad económica, como único medio para corregir las injusticias y la miseria que provoca el sistema económico (Castro, 2003: 11 y 118); lucha por una Constitución que le dé al hombre medio mejores garantías para su libertad política, para su trabajo, para su familia, para su vida pública $y$ privada $y$ para que pudiese vivir dignamente (Castro, 2003: 57-58); defiende la función social de la propiedad (Castro, 2003: 163); establece, que "el Estado tiene derecho a exigir impuestos para proveer los servicios públicos" (Castro, 2003: 100). En suma, considera que el Estado debe fomentar la producción nacional y el más adecuado reparto de la riqueza.

A la distancia, me parece, que el Constituyente Facio tenía muy claro que había que ayudar a la empresa privada, generadora de la riqueza nacional, para que el Estado pudiera hacer justicia social. Más claro, como diría el pueblo, "que sin cacao no se puede hacer chocolate".

\section{UNA CONSTITUCIÓN DE CONSENSO Y ABIERTA AL CAMBIO}

No obstante la claridad ideológica de Rodrigo Facio y a pesar de que se ha dicho que la Constitución aprobada en 1949 no es una Constitución neutral, desde ningún punto de vista se puede inferir que la nuestra sea la Constitución de un partido político. Rodrigo Facio condenaba la intolerancia, el mesianismo político y los dogmatismos de toda clase. Sus luchas fueron por una Constitución dirigida a un pueblo, como decía el Constituyente Facio, "con puntos de vista fundamentalmente diferentes" (Castro, 2003: 66). Su legitimidad se basa en la capacidad que tuvieron los constituyentes de 1949, para acoger por consenso los principios y los valores fundamentales de la sociedad costarricense, representados en la Asamblea Nacional Constituyente.

Se podría decir, que la Constitución de Costa Rica es una Constitución de Consenso, una Constitución que se dicta para un pueblo con puntos de vista fundamentalmente diferentes, en la que se definen los valores superiores del ordenamiento democrático constitucional, se organizan las instituciones del Estado y se garantizan los derechos fundamentales, pero de una manera flexible y abierta al cambio.

En sus alocuciones, Facio deja claro que se opone al formalismo $y$ la rigidez del ordenamiento jurídico. Enfatiza, que nada es definitivo ni incuestionable $y$ menos en el mundo del derecho (Castro, 2003: 30); por eso, se opone a la existencia de normas pétreas o contenidos constitucionales inmodificables. Para él, me parece, el derecho no solo responde a las normas y preceptos dictados por el legislador constituyente, sino a un cuerpo que crece, cambia, se moldea y reinterpreta de manera dinámica, como causa de los avatares de la vida y el paso del tiempo, tal y como testimonia nuestra Carta Fundamental después de 62 años de existencia.

\section{CONCLUSIÓN}

En suma, Rodrigo Facio, fue la figura central, el personaje estelar de la Asamblea Nacional Constituyente que dictó la Carta Fundamental que hoy nos rige. Tenía claro lo que era un Estado democrático y social de derecho; y cumplió a perfección su misión, con mucho trabajo, estudio, perseverancia y como un hábil negociador.

Sin duda alguna, fue el principal ideólogo de nuestra Constitución Política. Lo digo con énfasis, hoy, cuando la moda de nuestros 
líderes políticos es renegar de la ideología y decir que se es de centro, a pesar de que eso conduzca, a nada más que al estancamiento político, económico y social del país.

El pensamiento de Rodrigo Facio se reviste de una particular actualidad, en momentos cuando la democracia costarricense, como diría O' Donnell, no tiene un buen desempeño. La opinión generalizada es que los últimos gobiernos, dominados por el personalismo y el egocentrismo, han perdido el rumbo; no se están solucionado cuestiones básicas del desarrollo, como la pobreza y la desigualdad social; tampoco ha encarado, con valentía e ingenio, el problema relacionado con la inseguridad ciudadana, producto tanto de la criminalidad común como del lavado de dólares, el narcotráfico y el sicariato.

Hoy analistas y expertos son del criterio que como país, no sabemos lo que queremos y que no hay nadie, ni siquiera la Presidente, que nos pueda dar una luz para saber hacia dónde ir. Al contrario de lo que hizo Rodrigo Facio, no se quiere hablar de política y se reniega de la ideología. Un país que no define lo que quiere, es como un barco perdido en altamar.

De ahí que, posiblemente, con mucho pesar para Rodrigo Facio, si estuviera vivo, constataría que por no hablarse de política, el tamaño o la dimensión de las acciones públicas emprendidas por los últimos gobiernos, han estado determinadas por problemas coyunturales, como la necesidad de arreglar una rendija en un puente o la de juntar las piedras caídas de una carretera recién inaugurada.

Rodrigo Facio fue por mucho, la figura central en la Constituyente de 1949, pero no por calculador, timorato o populista. Fue la figura estelar porque con claridad y valentía, desde una visión social democrática del Estado, logró que la Constitución que nos rige tuviese como fin, al ser humano, su dignidad y los derechos fundamentales que le son inherentes.

\section{BIBLIOGRAFÍA}

Castro Vega, Óscar. Rodrigo Facio en la Constituyente de 1949. San José. Costa Rica: EUNED, 2003.

Laski, Harold. Los derechos humanos. San José. Editorial Universidad de Costa Rica, 1978: 7.

Monge Álvarez, Luis Alberto. "Prólogo". Rodrigo Facio en la Constituyente de 1949. En: Castro Vega, Óscar. San José. Costa Rica. EUNED, 2003: XV.

Fecha de ingreso: 05/09/2011 Fecha de aprobación: 14/10/2011 